\title{
Qualidade da dieta entre idosos com arranjo familiar diferenciado na região do Vale do Sinos, Rio Grande do Sul
}

\author{
Francine da Rocha Flores", Flávia Porto Wieck", João Carlos Jaccottet Piccoli"**; Maria Helena Weber ${ }^{* * * *}$
}

\section{Resumo}

O objetivo deste estudo descritivo, de corte transversal, é comparar a qualidade da dieta de idosos que residem sozinhos com idosos que residem na companhia de algum familiar. Os participantes eram frequentadores de grupos de convivência de terceira idade, de uma população de 431 idosos, com idade entre 60 e 79 anos. Foi selecionado o número total daqueles que residiam sozinhos, 136 indivíduos, mais os 136 primeiros da lista de amostragem, que residiam na companhia de algum familiar, resultando em uma amostra composta por 272 idosos. Como instrumento, utilizou-se o índice de alimentação saudável (IAS) além de medidas antropométricas e um questionário sociodemográfico. A idade média obtida foi 70,7 anos $( \pm 5,3)$, com $94,1 \%$ do sexo feminino. Dentre o total da amostra de idosos, a média do índice de massa corporal foi de $29,3 \mathrm{~kg} / \mathrm{m}^{2}( \pm 4,3)$, classificados, assim, como sobrepeso. O escore total do IAS encontrado no grupo de idosos que residem sozinhos foi 78,5 pontos $( \pm 11,8)$ e os idosos que residem com familiar obtiveram 77,5 pontos $( \pm$ $16,2)$, indicando necessidade de melhoria na dieta para ambos. Os achados revelaram que não houve diferença estatística da qualidade da dieta de idosos que residem sozinhos quando comparados com aqueles que residiam com a família.

Palavras-chave: Envelhecimento. Hábitos alimentares. Nutrição do idoso.

\section{Introdução}

A população está envelhecendo e estudos mostram que o número de pessoas idosas cresce em um ritmo maior do que o número de pessoas que nascem. No Brasil, o ritmo do crescimento da popu-

* Nutricionista. Universidade Feevale. Endereço para correspondência: Rua Arcedino Francisco da Conceição, 211. Bairro Lomba Grande, CEP 93490-500. Novo Hamburgo, RS. E-mail: francinefflores@hotmail.com

** Mestre em Gerontologia Biomédica pela Pontifícia Universidade Católica do Rio Grande do Sul. Especialista em Nutrição Clínica e Nutricionista pela Universidade do Vale do Rio dos Sinos. Universidade Feevale. E-mail: portofla@gmail.com

*** Doutor em Educação Física pela Ohio State University, USA. Professor aposentado da Escola Superior de Educação Física, Universidade Federal de Pelotas, Pelotas, RS. Ex-docente do Programa de Pós-Graduação em Diversidade Cultural e Inclusão Social, curso de Educação Física da Universidade Feevale, Novo Hamburgo, RS. E-mail: jebpiccoli@terra.com.br

**** Nutricionista. Especialista em Ciências do Esporte. Doutora em Bioquímica pela Universidade Federal do Rio Grande do Sul. Docente do curso de Nutrição da Universidade Feevale. E-mail: helenaweber@feevale.br

$\rightarrow$ http://dx.doi.org/10.5335/rbceh.v13i2.5687

Recebido em: 02/03/2016. Aceito em: 20/06/2016. 
lação idosa tem sido constante. Segundo a Pesquisa Nacional por Amostra de Domicílios 2009, o Brasil contava com uma população de 21 milhões de pessoas de 60 anos ou mais de idade (INSTITUTO BRASILEIRO DE GEOGRAFIA E ESTATÍSTICA, 2010; NERI; GUARIENTO, 2011).

A alimentação adequada e equilibrada e um bom estado nutricional estão associados a um envelhecimento saudável, que, combinado a outras medidas, aumenta a expectativa de vida com qualidade, podendo atuar como fator de prevenção e controle de várias doenças. No entanto, o envelhecimento é acompanhado de mudanças que podem alterar a ingestão alimentar e, como consequência, levar à desnutrição, que, quando não tratada de forma adequada, pode contribuir para o aparecimento e a evolução de enfermidades degenerativas e distúrbios associados ao processo de envelhecimento (MAGNONI; CUKIER; OLIVEIRA, 2010; NERI; GUARIENTO, 2011; SOUSA; MARQUES; VASCONCELOS, 2014).

O isolamento social, a depressão, a viuvez e as incapacidades são alguns dos principais fatores que alteram a ingestão alimentar do idoso, podendo apresentar como consequência a depleção de nutrientes essenciais para a manutenção da saúde e o controle de doenças (MAGNONI; CUKIER; OLIVEIRA, 2010; ASSUMPÇÃO et al., 2014). Tendo em vista as consequentes doenças crônicas e os efeitos da alimentação inadequada, conhecer e identificar os padrões dietéticos tornou-se indispensável. Para isso, foram desenvolvidos instrumentos dietéticos que determinam a qualidade da dieta dos indivíduos. Dentre os vários índices dietéticos, cabe destacar o índice de alimentação saudável ( $\mathrm{He}$ althy Eating Index), caracterizado por uma medida sintética da qualidade da dieta, que pode ser usada para avaliar e guiar a ingestão da dieta individual e populacional, promovendo a saúde e prevenindo doenças por meio de educação nutricional (ANDRADE; VOLP; NASCIMENTO, 2014).

Sendo assim, o objetivo geral do presente estudo foi comparar a qualidade da dieta de idosos que residem sozinhos com a de idosos que residem na companhia de algum familiar. Os objetivos específicos eram verificar as características da amostra, classificar o estado nutricional da amostra, conhecer o consumo de energia e macronutrientes e classificar a qualidade da dieta pelo índice de alimentação saudável (IAS).

\section{Metodologia}

Os indivíduos participantes foram selecionados em uma pesquisa multidisciplinar, descritiva, de corte transversal, conduzida por uma universidade, a qual intitulou-se Envelhecimento e qualidade de vida: um estudo em municípios selecionados da região do Vale dos Sinos, RS. Foram avaliados idosos de ambos os sexos com idades entre 60 e 79 anos. Os participantes eram frequentadores de grupos de convivência de terceira idade, os quais tinham uma população de 431 idosos. 
Por intermédio de um questionário sociodemográfico, foram selecionados 136 indivíduos que residiam sozinhos e outros 136 que residiam na companhia de algum familiar, resultando em uma amostra composta por 272 idosos. Os critérios para inclusão neste estudo foram: idade dos indivíduos (60 a 79 anos), estar residindo na companhia de algum familiar ou sozinho.

O período de coleta dos dados ocorreu de agosto de 2011 a abril de 2014 . A fim de se obter as características da amostra, aplicou-se um questionário sociodemográfico em que foram obtidas informações acerca de idade, gênero, grau de escolaridade, renda, tipo de moradia, anos de estudo, aposentadoria e com quem reside. Os dados antropométricos foram obtidos pelo uso de um estadiômetro para aferir a estatura e de uma balança antropométrica da marca Welmy ${ }^{\circledR}$ para verificar o peso corporal; para não comprometer ou interferir no resultado final, solicitou-se aos idosos que permanecessem apenas com as roupas íntimas e descalços. Para a avaliação da composição corporal e para classificar o estado nutricional, foi utilizado o índice de massa corporal (IMC), que é obtido dividindo-se o valor da massa corporal em quilogramas pelo da estatura, em metros, elevada ao quadrado $\left(\mathrm{kg} / \mathrm{m}^{2}\right)$.

Os pontos de corte adotados foram os propostos pela Organização Pan-americana de Saúde (Opas), direcionado à população idosa, que estabelece que resultados inferiores a $23 \mathrm{~kg} / \mathrm{m}^{2}$ são classificados como baixo peso, valores até $28 \mathrm{~kg} / \mathrm{m}^{2}$ consideram-se eutrofia, acima desse valor até $30 \mathrm{~kg} / \mathrm{m}^{2}$ será sobrepeso e resultados superiores são classificados como obesidade.

Para avaliar o consumo de energia e de macronutrientes, aplicou-se o instrumento recordatório alimentar de 24 horas (R24h) por meio de entrevista, na qual o idoso descreveu todas as refeições feitas no dia anterior à consulta nutricional, citando os alimentos ingeridos e suas quantidades. A análise da quantidade dos alimentos foi feita pela descrição de medidas caseiras. Para se ter uma maior veracidade das informações, foram utilizadas imagens ilustrativas de diversos utensílios e alimentos com seus pesos e medidas (álbum fotográfico).

Os dados alimentares de cada idoso foram cadastrados no software Dietwin Profissional $^{\circledR}$, com o objetivo de avaliar o consumo alimentar e, posteriormente, analisar determinados componentes do índice de alimentação saudável. O IAS é um instrumento de medida sumária do padrão alimentar, baseado no R24h, criado por Kennedy e colaboradores, constituído por dez componentes (cereais, hortaliças, frutas, leite, carne, gordura total, gordura saturada, colesterol, sódio e variedade da dieta), considera-se que para cada componente há uma pontuação, que varia de zero a dez, e um índice que varia de zero a cem (KENNEDY et al., 1995).

Ao final da análise, a classificação para a qualidade da dieta é dividida em três categorias. Quando a pontuação fica abaixo de 51 pontos, a dieta é classificada como "inadequada", quando pontua entre 51 e 80, é classificada como 
"necessita de melhorias", e quando a pontuação é maior de 80 e até 100 pontos, classifica-se a dieta como "saudável" (VOLP et al., 2010).

As variáveis quantitativas foram descritas por média e desvio padrão e as variáveis categóricas foram descritas por frequências absolutas e relativas. Para comparar médias, utilizaram-se os testes T de Student para amostras independentes ou o de análise de variância one-way (ANOVA one-way). Na comparação de proporções, os testes Qui-Quadrado de Pearson ou o exato de Fisher foram utilizados. O nível de significância adotado foi de $5 \%(\mathrm{p} \leq$ 0,05 ) e as análises foram realizadas no programa SPSS, versão 21.0.

A pesquisa foi submetida ao Comitê de Ética e Pesquisa da Universidade Feevale, tendo sido aprovada de acordo com o Processo no 4.06.01.10.1729. Após a concordância, todos os participantes assinaram o termo de consentimento livre e esclarecido.

\section{Resultados}

A amostra final do estudo foi constituída por 272 idosos, dos quais 136 residiam sozinhos, com idade média de 70,7 anos $( \pm 5,3)$, a grande maioria era do sexo feminino $(94,1 \%), 75 \%$ tinham o ensino fundamental incompleto, $87 \%$ contavam com renda familiar entre dois e cinco salários mínimos e $89 \%$ eram aposentados. A outra metade da amostra era de 136 idosos que morava com a família, desses, $94(69,1 \%)$ com filhos, $28(20,6 \%)$ com companheiro, 8 (5,9\%) com companheiro e filhos e $6(4,4 \%)$ com outros familiares (netos, irmãos). A idade média constatada foi de 68,6 anos $( \pm 5,0)$,maioria do sexo feminino $(80,9 \%)$, $70,6 \%$ tinham ensino fundamental incompleto, $69,9 \%$ contavam com renda familiar de dois a cinco salários mínimos e 84,6\% eram aposentados.

Quando comparados os grupos quanto às características sociodemográficas, houve diferença significativa quanto à idade $(p=0,001)$ e ao sexo $(p=0,002)$. Idosos que moravam sozinhos tinham idade superior e apresentavam maior proporção de mulheres quando comparados com os que residiam com a família. Em relação ao estado nutricional da amostra total de idosos, a classificação final do IMC foi: $5,1 \%$ baixo peso, $36,8 \%$ eutrofia, $16,5 \%$ sobrepeso e $41,5 \%$ obesidade. A média do IMC de ambos os grupos foi de $29,3 \mathrm{~kg} / \mathrm{m}^{2}( \pm 4,3)$, classificada como sobrepeso de acordo com a Opas (ORGANIZAÇÃO PAN-AMERICANA DA SAÚDE, 2003). A média do IMC dos idosos que residiam sozinhos foi de $29,5 \mathrm{~kg} / \mathrm{m}^{2}( \pm 4,4)$, classificada como sobrepeso, de acordo com a Opas. Os que residiam com familiares apresentaram a média de $29,1 \mathrm{~kg} / \mathrm{m}^{2}( \pm 4,1)$, obtendo a mesma classificação.

Ao serem analisados separadamente os resultados dos dois grupos, conforme a Figura 1, houve prevalência de obesidade em ambos, porém, notou-se que nos idosos que moravam sozinhos o índice de obesidade foi significativo, com $43,4 \%$, enquanto que no grupo dos idosos que residiam com familiar representou $39,7 \%$. 
Figura 1 - Estado nutricional dos idosos

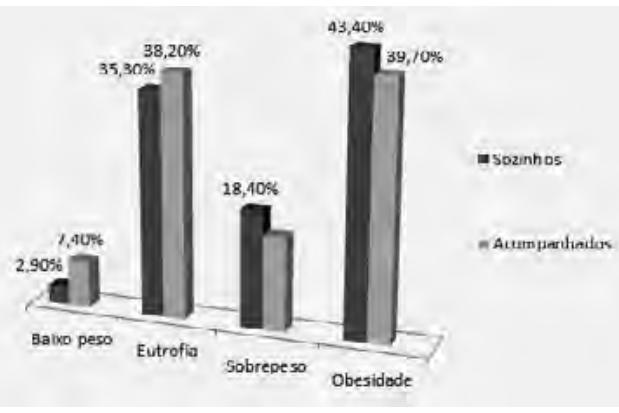

Fonte: elaborada pelos autores com base em coleta de dados.

A partir do recordatório de 24 horas, a análise do consumo alimentar da amostra total revelou uma média de consumo de calorias ingeridas durante o dia abaixo do recomendado pelas Dietary Reference
Intakes (DRIs), que varia de 1.873 a 2.054 kcal, de acordo com sexo e idade. Já a distribuição dos macronutrientes encontra-se dentro do recomendado pelas DRIs para idosos. A recomendação diária de proteína por $\mathrm{kg} /$ peso, de acordo com as DRIs, é de 0,9 a $1,1 \mathrm{~g}$ de proteína por $\mathrm{kg} /$ peso, o que demonstra que os idosos de ambos os grupos estão consumindo quantidade acima do preconizado. Quando avaliado o consumo alimentar, os idosos que residiam sozinhos apresentaram um consumo de proteína, em g/kg, significativamente mais elevado quando comparados aos idosos que residiam com a família (Tabela 1). Quanto aos demais macronutrientes, as diferenças não foram estatisticamente significativas.

Tabela 1 - Consumo alimentar na amostra total e conforme arranjo familiar

\begin{tabular}{l|r|r|r|r}
\hline \multicolumn{1}{c|}{ Variáveis } & \multicolumn{1}{c|}{$\begin{array}{c}\text { Amostra total } \\
(\mathrm{n}=272)\end{array}$} & $\begin{array}{c}\text { Reside sozinho } \\
(\mathrm{n}=136)\end{array}$ & $\begin{array}{c}\text { Reside com família } \\
(\mathrm{n}=136)\end{array}$ & $\mathrm{P}$ \\
\hline VET $^{*}$ & $1376 \pm 688$ & $1395 \pm 865$ & $1357 \pm 447$ & 0,653 \\
CHO (\%) & $56,3 \pm 10,3$ & $56,4 \pm 10,9$ & $56,1 \pm 9,7$ & 0,834 \\
PTN $(\%)^{* *}$ & $17,4 \pm 6,3$ & $17,5 \pm 7,2$ & $17,3 \pm 5,1$ & 0,733 \\
PTN $(\mathrm{g} / \mathrm{kg})^{* *}$ & $1,41 \pm 0,83$ & $1,55 \pm 0,76$ & $1,27 \pm 0,87$ & 0,005 \\
LIP (\%) & $26,4 \pm 8,4$ & $26,2 \pm 8,9$ & $26,6 \pm 8,0$ & 0,705 \\
\hline
\end{tabular}

Fonte: elaborada pelos autores com base em coleta de dados.

Nota: * Variável apresentada em forma de média (desvio padrão); * Variáveis apresentadas em forma de frequência (percentual).

Outra etapa do estudo analisou o consumo alimentar dos participantes e pontuá-lo de acordo com o índice de alimentação saudável. Observando-se os itens do IAS, deve-se chamar a atenção para a média de pontuação referente ao consumo de cereais, hortaliças e leite de ambos os grupos, sendo uma pontuação baixa em comparação aos outros itens. $\mathrm{Na}$ avaliação da qualidade da dieta conforme a residência, houve diferença significativa entre os grupos quanto aos cereais $(\mathrm{p}=0,010)$, à carne $(\mathrm{p}=0,043)$ e à gordura saturada $(\mathrm{p}=0,018)$. Idosos que residem sozinhos têm pontuação superior nos grupos de cereais e gordura saturada, mas inferior em carne. A Tabela 2 demonstra os valores das porções consumidas ao dia e a pontuação correspondente. 
Tabela 2 - Pontuação dos componentes e classificação do IAS da amostra total, segundo arranjo familiar

\begin{tabular}{l|r|r|r|r}
\hline \multicolumn{1}{c|}{ IAS* $^{*}$} & $\begin{array}{c}\text { Amostra total } \\
(n=272)\end{array}$ & $\begin{array}{c}\text { Reside sozinho } \\
(n=136)\end{array}$ & $\begin{array}{c}\text { Reside com } \\
\text { família }(n=136)\end{array}$ & $P$ \\
\hline Grupo 1 - Cereais & $6,93 \pm 2,62$ & $7,34 \pm 2,37$ & $6,52 \pm 2,80$ & 0,010 \\
Grupo 2 - Hortaliças & $4,33 \pm 3,42$ & $4,34 \pm 3,16$ & $4,31 \pm 3,69$ & 0,953 \\
Grupo 3 - Frutas & $7,40 \pm 3,53$ & $7,30 \pm 3,68$ & $7,50 \pm 3,38$ & 0,643 \\
Grupo 4 - Leite & $6,25 \pm 4,23$ & $6,07 \pm 4,25$ & $6,43 \pm 4,21$ & 0,480 \\
Grupo 5 - Carne & $9,04 \pm 2,96$ & $8,68 \pm 3,40$ & $9,40 \pm 2,38$ & 0,043 \\
Grupo 6 - Gordura total (\%) & $9,06 \pm 2,21$ & $9,06 \pm 2,40$ & $9,05 \pm 2,02$ & 0,960 \\
Grupo 7 - Gordura saturada (\%) & $8,59 \pm 2,82$ & $8,99 \pm 2,47$ & $8,18 \pm 3,10$ & 0,018 \\
Grupo 8 - Colesterol (mg) & $9,15 \pm 2,49$ & $9,31 \pm 2,32$ & $9,00 \pm 2,65$ & 0,313 \\
Grupo 9 - Sódio (mg) & $8,82 \pm 2,25$ & $8,98 \pm 2,02$ & $8,66 \pm 2,46$ & 0,249 \\
Grupo 10 - Variedade & $8,38 \pm 2,20$ & $8,45 \pm 2,13$ & $8,31 \pm 2,28$ & 0,607 \\
IAS total & $78,0 \pm 14,2$ & $78,5 \pm 11,8$ & $77,5 \pm 16,2$ & 0,534 \\
\hline
\end{tabular}

Fonte: elaborada pelos autores com base em coleta de dados.

Nota: "Variável apresentada em forma de média \pm DP.

O escore total do IAS encontrado no grupo de idosos que residiam sozinhos e $o$ encontrado no grupo daqueles que moravam com familiares obteve uma pontuação semelhante, o que indica necessidade de melhoria na dieta para os indivíduos de ambos os grupos. Em relação à classificação do IAS, metade dos idosos que residia sozinhos apresentaram dieta saudável, resultado semelhante ao grupo de idosos que residia com a família, em que pouco menos da metade apresentou esse resultado. Conforme a Figura 2, é possível conhecer a classificação geral dos grupos.
Figura 2 - Classificação do IAS conforme arranjo familiar

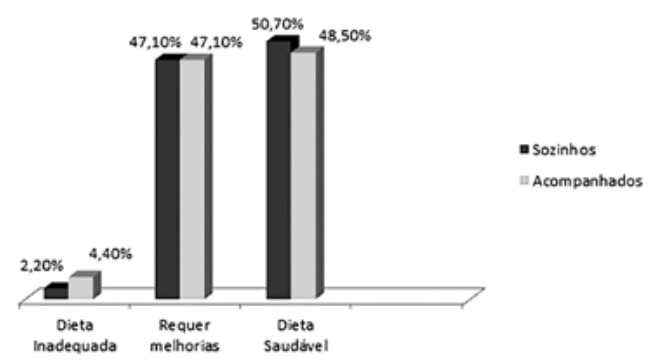

Fonte: elaborada pelos autores com base em coleta de dados.

Quando comparados os sexos (Tabela 3), os homens apresentaram melhor qualidade da dieta tanto no grupo leite $(\mathrm{p}=0,015)$ quanto no grupo carne $(\mathrm{p}=0,043)$. Na classificação do IAS, $61,8 \%$ dos homens pontuaram e obtiveram dieta adequada. No grupo feminino, $50,4 \%$ obtiveram pontuação do IAS para 
dieta que necessita de melhorias. Outra característica é o consumo de hortaliças, em que os homens obtiveram uma pontuação maior em comparação às mulheres, porém, ambas as pontuações foram baixas. Na classificação do IAS, os homens apresentaram uma dieta mais saudável (Tabela 3).

Tabela 3 - Qualidade da dieta de acordo com o sexo

\begin{tabular}{|c|c|c|c|}
\hline $\mathrm{IAS}^{*}$ & $\begin{array}{l}\text { Homens } \\
(n=34)\end{array}$ & $\begin{array}{l}\text { Mulheres } \\
(n=238)\end{array}$ & $P$ \\
\hline Grupo 1 - Cereais & $6,73 \pm 2,73$ & $6,96 \pm 2,61$ & 0,630 \\
\hline Grupo 2 - Hortaliças & $5,03 \pm 3,46$ & $4,23 \pm 3,41$ & 0,208 \\
\hline Grupo 3 - Frutas & $7,88 \pm 3,09$ & $7,33 \pm 3,59$ & 0,396 \\
\hline Grupo 4 - Leite & $7,78 \pm 3,73$ & $6,03 \pm 4,26$ & 0,015 \\
\hline Grupo 5 - Carne & $9,70 \pm 1,74$ & $8,95 \pm 3,08$ & 0,042 \\
\hline Grupo 6 - Gordura total (\%) & $9,04 \pm 2,24$ & $9,06 \pm 2,22$ & 0,971 \\
\hline Grupo 7 - Gordura saturada (\%) & $8,85 \pm 2,36$ & $8,55 \pm 2,88$ & 0,578 \\
\hline Grupo 8 - Colesterol (mg) & $9,16 \pm 2,53$ & $9,15 \pm 2,49$ & 0,994 \\
\hline Grupo 9 - Sódio (mg) & $8,20 \pm 2,66$ & $8,91 \pm 2,18$ & 0,088 \\
\hline Grupo 10 - Variedade & $8,08 \pm 2,64$ & $8,43 \pm 2,14$ & 0,398 \\
\hline IAS total & $79,1 \pm 14,8$ & $77,8 \pm 14,1$ & 0,628 \\
\hline Classificação IAS** & & & 0,022 \\
\hline Dieta inadequada** & $3(8,8)$ & $6(2,5)$ & \\
\hline Dieta necessita de melhorias ${ }^{\star *}$ & $10(29,4)$ & $120(50,4)$ & \\
\hline Dieta saudável ${ }^{\star \star}$ & $21(61,8)$ & $112(47,1)$ & \\
\hline
\end{tabular}

Fonte: elaborada pelos autores com base em coleta de dados.

"Variável apresentada em forma de média (desvio padrão).

${ }^{* * *}$ Variáveis apresentadas em forma de frequência (percentual).

\section{Discussão}

A amostra total do presente estudo foi composta em sua maioria por indivíduos do sexo feminino, o que é um fato comum em estudos populacionais. Isso, possivelmente, ocorreu devido ao número inferior de homens nos grupos de convivência de idosos incluídos na população estudada (LIMA; BUENO, 2009). A renda mensal média dos idosos de ambos os grupos foi de dois a cinco salários mínimos, resultado semelhante ao estudo de Mendonça, Relvas e Correa (2010), que teve como objetivo estudar as características do consumo alimentar dos idosos no Brasil.

Em relação ao estado nutricional, o presente estudo obteve uma média de classificação de sobrepeso de acordo com a classificação da Opas. Resultados semelhantes foram encontrados por Al- 
termann e colaboradores (2011), que realizaram um estudo para avaliar o estado nutricional de uma amostra composta por 34 idosos e obtiveram uma média do índice de massa corporal de $28,74 \mathrm{~kg} / \mathrm{m}^{2}$ $( \pm 6,29)$. Também Sonati e colaboradores (2014) encontraram resultado semelhante em estudo cujo objetivo era identificar as diferenças entre as percepções na qualidade de vida de aultos e idosos, ambos praticantes de atividade física, em uma amostra composta por 56 adultos e 61 idosos, na qual este último grupo, tinha em média um índice de massa corporal de $27,46 \mathrm{~kg} / \mathrm{m}^{2} \pm 4,20$.

Neumann e colaboradores (2014), em estudo que teve como objetivo avaliar o estado nutricional e associá-lo à prevalência de doenças crônicas não transmissíveis, em uma amostra de 112 idosos, encontraram uma maioria $(57,1 \%)$ de obesos/excesso de peso e uma minoria $(15,2 \%)$ com baixo peso/desnutrição, assim como no estudo de Nascimento e colaboradores (2011), que encontraram um resultado de $45,5 \%$ com excesso de peso e $13,6 \%$ com baixo peso.

Os dados de consumo alimentar encontrados revelaram que os valores de calorias ingeridas por dia estão abaixo do recomendado pelas DRIs. Valores também verificados por Menezes e Marucci (2012), que constataram um consumo com média de 1.316,3 kcal ( \pm 509$)$ em 458 idosos estudados. Outro estudo que obteve o mesmo resultado foi desenvolvido por Domene e colaboradores (2014), que concluiu a baixa ingestão calórica de sua amostra de 1.510 idosos, com consumo diário de 1.623,9 kcal.
O consumo de proteínas por $\mathrm{kg} / \mathrm{peso}$ dos idosos de ambos os grupos do presente estudo estava acima do preconizado. Em outra pesquisa, com uma amostra de 22 idosos, foi possível verificar que $31,82 \%$ ingerem menos que o ideal de proteínas diariamente, e que $36,36 \%$ consomem acima do recomendado (LEITE; BARATTO, 2014).

Em relação ao índice de alimentação saudável, a média de pontos da amostra total do presente estudo foi de 78,0 $( \pm 14,2)$. Na variável sexo, obteve-se 79,1 $( \pm 14,8)$ para homens e $77,8( \pm 14,1)$ para mulheres, ambos os casos classificados como necessitando de melhorias na dieta. Tal resultado foi encontrado, também, por Assumpção e colaboradores (2014), que apresentaram uma pesquisa com 1.509 idosos, cujo objetivo foi verificar a qualidade da dieta e os fatores associados. Nesse estudo, foi utilizado como instrumento o índice de qualidade da dieta revisado, proposto por Previdelli e colaboradores (2011). A pontuação encontrada da amostra total foi 62,4 (IC95\%: 61,7-63,1), sendo 61,8 (IC95\%: 60,0-63,6) para homens e 62,2 (IC95\%: 61,5-63,7) para mulheres, classificando-se também como dieta que necessita de melhorias.

No presente estudo, obteve-se 3,3\% da amostra total indicando dieta inadequada, $47,8 \%$ dieta que necessita de melhorias e 48,9\% indicando dieta saudável. O mesmo resultado apresentaram Abreu e colaboradores (2013), em um estudo para verificar a associação entre a baixa renda e a qualidade da dieta de trinta idosos participantes de um grupo de terceira idade, apresentou 3,3\% de 
indivíduos com dieta inadequada e $66,7 \%$ com dieta saudável, de acordo com $o$ índice de qualidade da dieta revisado.

Como instrumento para mensurar a qualidade da dieta, encontra-se disponível, também, o índice de alimentação saudável adaptado para a população brasileira (IASad) por Mota e colaboradores (2008). Esse instrumento foi utilizado em um estudo de Leite e colaboradores (2013) que investigou a alimentação de 56 idosos com diferentes condições dentárias, dos quais, dez obtiveram boa qualidade, dez, má qualidade e 36 precisando de melhorias na dieta.

Quando analisado os componentes do índice de alimentação saudável, observa-se que o consumo de frutas, hortaliças, cereais e leite e derivados, no presente estudo, atingiu pontuação baixa quando comparados com os outros componentes do índice. As porções são pontuadas conforme as indicações da pirâmide alimentar e, assim, essa amostra não alcançou a recomendação desses grupos. Dados semelhantes foram descritos na pesquisa de Malta, Papini e Corrente (2013), que tinha o objetivo de avaliar a qualidade da dieta de uma população de 73 idosos, utilizando o IASad. Os autores verificaram que o consumo de frutas, hortaliças, cereais, leite e derivados teve uma baixa pontuação.

Alguns achados limitaram a discussão dos resultados, pois houve controvérsias sobre o consumo de proteínas ( $\mathrm{g} / \mathrm{kg}$ ) entre os grupos. Aqueles que residiam sozinhos apresentaram quantidades superiores quando comparados aos da família. Uma suposição seria a de que apesar de a renda mensal ser a mesma nos dois grupos, ela era "diluída" pela aquisição dos gêneros alimentícios no caso dos que residiam com a família diferentemente daqueles que moravam sozinhos. Essa restrição também foi encontrada ao se buscar na literatura estudos que analisavam o consumo alimentar de idosos que residiam em diferentes arranjos familiares, dificultando a discussão dos resultados do presente estudo. Outra questão que deve ser ressaltada é que o instrumento utilizado para o cálculo do IAS foi o recordatório de 24 horas, pois, mesmo com o apoio do álbum fotográfico para estimativa de quantidades, há dependência da memória do consumo alimentar do dia anterior.

\section{Conclusão}

O objetivo do estudo foi verificar se haveria diferença na classificação de qualidade da dieta ao comparar idosos que moravam sozinhos com aqueles que residiam com algum familiar. Tal fato não foi comprovado, uma vez que a pontuação do índice de qualidade da dieta foi semelhante entre os dois grupos. Diversas investigações referentes ao consumo alimentar de idosos abordam a relação entre monotonia alimentar e qualidade da dieta. Esse foi o eixo norteador desta pesquisa, pois, ao aplicar o recordatório alimentar, percebeu-se o quão limitado era o consumo dos idosos que moravam sozinhos.

Assim, esta investigação mostrou que a pontuação referente à variedade da dieta entre os grupos foi igual, indi- 
cando que tanto os idosos que residiam sozinhos quanto os que residiam com a família tinham à sua disposição o mesmo número de alimentos. Com isso, deve-se considerar que alguns componentes do IAS podem subestimar ou superestimar o consumo, como no caso da variedade da dieta, na qual não se diferencia o consumo de alimentos saudáveis dos que não são saudáveis, não refletindo necessariamente a qualidade da dieta.

\section{Quality of diet among elderly with different residential status in the Vale do Sinos region, state of Rio Grande do Sul}

\section{Abstract}

The objective of this study was to compare the quality of the diet for the elderly who lived alone to the ones who lived with any family member. The subjects participated of companionship centers/groups with a population of 431 elder people, aging between 60 and 79 . The number of those who lived alone were selected, being 136 individuals, plus the first 136 of the sample list who lived in the company of a family member, resulting in a sample composed of 272 persons. The data were collected through the "Healthy eating index" $(\mathrm{HEI})$, as well as the anthropometric measurements and a sociodemographic questionnaire. The average age was 70,7 $\pm 5,3$ years, being $94,1 \%$ female. Among the total sample of elderly, the average IMC was $29,3 \pm 4,3 \mathrm{~kg} / \mathrm{m}^{2}$, classified as overweight. The total score of $\mathrm{HEI}$ found in the group of old people who live alone was $78,5 \pm 11,8$ points and the ones who live with any family member obtained $77,5 \pm$ 16,2 points, indicating the necessity of diet improvement for both groups. The findings show that there wasn't any statistical difference in the quality of the diet of old persons who live alone comparing to the ones who live in family.

Keywords: Aging. Elderly nutrition. Food habits.

\section{Referências}

ABREU, I. C. M. E. et al. Baixa renda entre os idosos participantes da terceira idade está associada à qualidade da dieta. Revista de Alimentos e Nutrição, Araraquara (SP), v. 24, n. 3, p. 349-357, jul./set. 2013.

ALTERMANN, C. S. et al. Avaliação antropométrica em idosos participantes do dia do desafio em Santa Maria/RS. Revista Contexto e Saúde, Ijuí (RS), v. 10, n. 20, p. 733-736, jan./jun. 2011.

ANDRADE, H. D. R.; VOLP, A. C. P.; NASCIMENTO, R. F. Avaliação da qualidade da dieta pelo índice de alimentação saudável e suas variações: uma revisão bibliográfica. Nutrición Clínica Y Dietética Hospitalaria, Madrid, v. 34, n. 2, p. 88-96, 2014.

ASSUMPÇÃO, D. et al. Qualidade da dieta e fatores associados entre idosos: estudo de base populacional em Campinas, São Paulo, Brasil. Caderno de Saúde Pública, Rio de Janeiro (RJ), v. 30, n. 8, p. 1.680-1.694, ago. 2014.

DOMENE, S. M. A. et al. Ingestão de minerais e fitatos: indicadores para o monitoramento de risco nutricional. Revista Visa em Debate, São Paulo (SP), v. 2, n. 4, p. 69-75, 2014.

INSTITUTO BRASILEIRO DE GEOGRAFIA E ESTATÍSTICA. Síntese de indicadores sociais: uma análise das condições de vida da população brasileira 2010. Brasília: Ministério do Planejamento, Orçamento e Gestão, 2010. Disponível em: <www.ibge.gov.br/ home/estatistica/populacao/condicaodevida/ indicadoresminimos/sinteseindicsociais2010/ SIS_2010.pdf>. Acesso em: 19 fev. 2016. 
KENNEDY, Kennedy, E. T. et al. The Healthy Eating Index: design and applications. Journal of the American Dietetic Association, v. 95, n. 10, p. 1.103-1.109, Oct. 1995.

LEITE, A. P. D. et al. Aspectos alimentares em idosos com diferentes condições dentárias. Revista Brasileira de Qualidade de Vida, Ponta Grossa (PR), v. 5, n. 3, p. 48-58, jul./set. 2013.

LEITE, S.; BARRATTO, C.; SILVA, R. Consumo de cálcio e risco de osteoporose em uma população de idosos. Revista Brasileira de Obesidade, Nutrição e Emagrecimento, São Paulo, v. 8, n. 48, p. 165-174, nov./dez. 2014.

LIMA, L. C. V.; BUENO, C. M. L. B. Envelhecimento e gênero: a vulnerabilidade de idosas no Brasil. Revista de Saúde e Pesquisa, Maringá (PR), v. 2, n. 2, p. 273-280, maio/ ago. 2009.

MAGNONI, D.; CUKIER, C.; OLIVEIRA, P. A. Nutrição na terceira idade. 2. ed. São Paulo: Sarvier, 2010.

MALTA, M. B.; PAPINI, S. J.; CORRENTE, J. E. Avaliação de alimentação de idosos de município paulista - aplicação do índice de alimentação saudável. Revista Ciência \& Saúde Coletiva, Rio de Janeiro (RJ), v. 18, n. 2, p. 377-384, 2013.

MENDONÇA, P. S. M.; RELVAS, K.; CORREA, S. R. A. Estudo do comportamento de consumidores idosos no Brasil: alimentos preferidos, perfil do consumidor e contexto de consumo. Revista Ciência Administrativa, Fortaleza (CE), v. 16, n. 2, p. 529-543, jul./dez. 2010.

MENEZES, T. D.; MARUCCI, M. F. M. Valor energético total e contribuição percentual de calorias por macronutrientes da alimentação de idosos domiciliados em Fortaleza - CE. Revista Associação Medica Brasileira, São Paulo (SP), v. 58, n. 1, p. 33-40, 2012.

MOTA, J. F. et al. Adaptação do índice de alimentação saudável ao guia alimentar da população brasileira. Revista de Nutrição, Campinas (SP), v. 21, n. 5, p. 545-552, set./ out. 2008.
NASCIMENTO, C. M. et al. Estado nutricional e condições de saúde da população idosa brasileira: revisão da literatura. Revista Médica, Belo Horizonte (MG), v. 21, n. 2, p. 174-180, 2011.

NERI, A. L.; GUARIENTO, M. E. Fragilidade, saúde e bem-estar em idosos. Campinas: Alíneia, 2011.

NEUMANN, B. et al. Associação entre o estado nutricional e a prevalência de doenças crônicas não transmissíveis em idosos residentes no município de Roca Sales-RS. Revista Brasileira de Ciências do Envelhecimento Humano, Passo Fundo (RS), v. 11, n. 2, p. 166-177, maio/ago. 2014.

ORGANIZAÇÃO PAN-AMERICANA DA SAÚDE. Saúde, bem-estar e envelhecimento - o Projeto Sabe no município de São Paulo: uma abordagem inicial. Brasília: Opas, 2003.

PREVIDELLI, N. A. et al. Índice da qualidade da dieta revisado para população brasileira. Revista de Saúde Pública da USP, São Paulo (SP), v. 45, n. 4, p. 794-798, 2011.

SONATI, J. G. et al. Análise comparativa da qualidade de vida de adultos e idosos envolvidos com a prática regular de atividade física. Revista Brasileira de Geriatria e Gerontologia, Rio de Janeiro (RJ), v. 17, n. 4, p. 731-739, 2014.

SOUSA, M. O.; MARQUES, M. P.; VASCONCELOS, S. M. Análise de cardápios oferecidos a idosos residentes em instituição de longa permanência. Revista Interdisciplinar, Teresina (PI), v. 7, n. 1, p. 1-7, jan./mar. 2014.

VOLP, A. C. P. et al. Índices dietéticos para avaliação da qualidade de dietas. Revista de Nutrição, Campinas (SP), v. 23, n. 2, p. 281-295, mar./abr. 2010. 